\title{
Race Against Time: Addressing the Unmet Needs of Patients with HER2-Positive Metastatic Breast Cancer
}

\author{
Authors: \\ Katarzyna Rygiel \\ Department of Family Practice, Medical University of Silesia (SUM), Zabrze, Poland \\ *Correspondence to kasiaalpha@yahoo.co.uk \\ Disclosure: \\ The author has declared no conflicts of interest. \\ Received: \\ 04.02 .20 \\ Accepted: \\ 21.04.20 \\ Keywords: \\ Antibody-drug conjugates (ADC), brain metastases, breast cancer (BC), human \\ epidermal growth factor receptor-2 (HER2)-positive metastatic breast cancer, \\ tyrosine kinase inhibitors (TKI).
}

Citation:

EMJ Oncol. 2020;8[1]:96-104.

\section{Abstract}

Over the last 20 years, there has been remarkable progress in the development of the therapies for human epidermal growth factor receptor-2 (HER2)-positive breast cancer (BC). Targeted treatment agents, such as trastuzumab, pertuzumab, lapatinib, and trastuzumab emtansine (T-DM1), are currently recommended as key components in the standard of care regimens for patients with HER2positive BC. However, some patients still develop disease progression despite using such therapies. Since brain metastases present an urgent unmet need in many women with HER2-positive BC, clinical studies focussing on novel strategies in this field are a high priority.

This brief overview outlines some recent results from relevant clinical trials, such as HER2CLIMB, SOPHIA, and DESTINY-BreastO1, in patients with HER2-positive metastatic BC, highlighting beneficial effects and safety issues of tucatinib, margetuximab, and trastuzumab deruxtecan (T-DXd), respectively. New research directions for the applications of these medications in combination with established treatment regimens are outlined. This article presents some insights into the potential transformation of clinical management and provides reasonable hope and encouragement to both the afflicted patients and their treatment teams.

\section{INTRODUCTION}

Breast cancer (BC), which is the most prevalent malignancy in women worldwide, is a heterogeneous disease, in which human epidermal growth factor receptor-2 (HER2) has a particular impact on the disease course, therapeutic response, and patient outcomes. The HER2 oncogene (termed HER2, HER2/neu, or ERBB2) is located on chromosome $17^{2}$ and its

main function is to encode the transmembrane receptor tyrosine kinase. ${ }^{3}$ HER2 gene amplification or overexpression (present in approximately $20 \%$ of all BC) has been related to tumour cell proliferation and invasion, causing local disease progression and distant metastases. ${ }^{4}$ In comparison to HER2-negative breast tumours, HER2-positive BC are characterised by aggressive behaviour and poor response to standard chemotherapy $(\mathrm{CHT})$ regimens. ${ }^{5}$ The expansive 
tumour behaviour resulting in poor patient outcomes had to date been devastating, until the era of anti-HER2-directed therapies. ${ }^{6}$

In fact, targeted anti-HER2 therapies, as the key strategies for HER2-positive BC (both early and advanced/metastatic stages), have altered the management and prognostic horizons for numerous women with HER2-positive BC. ${ }^{6}$ In particular, trastuzumab, a monoclonal IgG1 class humanised murine antibody, which binds to the extracellular domain of the HER2 transmembrane receptor; and lapatinib, a small-molecule tyrosine kinase inhibitor (TKI), which targets HER1 and HER2, have led to a therapeutic breakthrough in HER2-positive BC. ${ }^{6}$

Subsequently, pertuzumab has shown substantial benefits in the HER2-positive advanced and metastatic BC setting when added to trastuzumab-based therapy. It is a humanised monoclonal antibody that binds to HER2 on the extracellular domain II (a different domain than trastuzumab), preventing homoand heterodimer formations, and blocking the heterodimers HER2/HER3. ${ }^{6}$ Similarly, trastuzumab emtansine (T-DM1), an antibodydrug conjugate (ADC), which contains trastuzumab linked to a maytansine derivative (DM1) (a potent antimitotic agent binding to microtubules), has been added to the therapeutic armamentarium in this patient population. ${ }^{6}$

At present, in the first-line treatment for patients with HER2-positive metastatic BC, a dual anti-HER2 blockade (with trastuzumab and pertuzumab) plus CHT (a taxane) is recommended. T-DM1 (an ADC) is recommended for second-line treatment, and lapatinib or neratinib (a TKI) plus capecitabine or trastuzumab plus capecitabine or lapatinib for third-line or beyond. ${ }^{7}$ Although trastuzumab, pertuzumab, lapatinib, neratinib, and T-DM1 represent effective anti-HER2 agents, some patients still develop BC progression and subsequently have very limited treatment options. ${ }^{6,7}$ In particular, brain metastases present an urgent unmet need in many women with HER2-positive BC, and thus, clinical trials exploring novel treatment strategies in this area remain a high priority. ${ }^{8}$

This overview presents findings from several recent clinical studies, such as HER2CLIMB, SOPHIA, and DESTINY-BreastO1, in patients with
HER2-positive metastatic BC, highlighting the beneficial effects and safety issues of tucatinib, margetuximab, and trastuzumab deruxtecan (T-DXd). ${ }^{9-11}$ In addition, new perspectives for using these medications in combination with established treatment regimens, which may renew clinical practice strategies, are briefly discussed. This article also provides some insights into the potential transformation of clinical management, giving reasonable hope and encouragement to both the afflicted patients and their treatment teams.

\section{BRAIN METASTASES: THE GROWING CHALLENGE IN PATIENTS WITH HER2- POSITIVE BREAST CANCER}

About $50 \%$ of patients with HER2-positive BC develop central nervous system (CNS) metastases during their $\mathrm{BC}$ course. Because this is an unmet medical need, attempts to provide systemic therapies that can penetrate the blood-brain barrier are critically important. ${ }^{8}$ Unfortunately, the incidence of CNS metastases in patients with HER2-positive BC has increased as the targeted therapies have recently extended the patient survival. ${ }^{8}$ At this point, conventional therapy for brain metastases includes surgery, radiation therapy, and some systemic HER2-targeted medications, such as TKI and CHT. Because surgery and radiation therapy for the treatment of CNS metastases have been associated with serious adverse effects, targeted therapies have been considered as an important option for this treatment group. ${ }^{8}$

Currently, there are no specific systemic treatments for patients with metastatic BC and CNS metastases, and therefore, there is an urgent need to explore novel therapies in this patient population. Unfortunately, different anti-HER2 agents (e.g., trastuzumab, pertuzumab, and T-DM1), because of their molecular structure and size, cannot cross the blood-brain barrier, and thus their role in the treatment of brain metastases has been limited.12 In contrast, small molecules, such as TKI (e.g., lapatinib or neratinib), represent better options to reach therapeutic levels within the CNS structures..$^{13}$ For instance, lapatinib (a reversible $\mathrm{TKI}$ ) has been studied as a targeted therapy for CNS metastases in patients with HER2-positive metastatic BC after progression on the first-line trastuzumab-based treatment.13 
Similarly, neratinib (an irreversible pan-HER [HER1, HER2, and HER4] and EGFR TKI), which additionally blocks PI3K/AKT and MAPK signalling pathways after HER2 receptor activation, has also been investigated as a therapy for CNS metastases in this patient population. ${ }^{13}$

It should be highlighted that the SUMMIT basket, a Phase II, single-arm trial, has been exploring the combination of endocrine therapy (ET), a monoclonal antibody, and neratinib, in heavily pretreated patients with HER2-positive metastatic BC (whose tumours harbour HER2 mutations).${ }^{14}$ Based on the preliminary results of the SUMMIT trial, neratinib will be considered for use in combination with capecitabine in patients with HER2-positive $\mathrm{BC}$ in the third-line, and beyond, metastatic setting, especially in the management of CNS metastases. ${ }^{14}$ In fact, data from the SUMMIT trial, such as the objective response rate of $53 \%$ and the progression- free survival (PFS) of 10 months, has been encouraging. Although diarrhoea was the most common adverse effect reported in the SUMMIT study, it was effectively managed by using antidiarrhoeal agents. ${ }^{14}$

\section{TUCATINIB: A NEW PERSPECTIVE FOR PATIENTS WITH BREAST CANCER AND BRAIN METASTASES}

Tucatinib is a highly selective TKI for the kinase domain of HER2. ${ }^{9}$ With regard to the mechanism of action, and in contrast to other TKI (which inhibit both HER2 and EGFR), tucatinib mostly spares the EGFR and blocks mainly the HER2 component. $^{9}$ Because of this selectivity, the gastrointestinal adverse effects (e.g., diarrhoea) and skin rash are reduced in patients. ${ }^{9}$

Table 1: A comparison between the tyrosine kinase inhibitors tucatinib, neratinib, and lapatinib.

\begin{tabular}{llll}
\hline TKI name & Tucatinib & Neratinib & Lapatinib \\
\hline TKI type & A selective HER2 TKI & $\begin{array}{l}\text { An irreversible pan-HER TKI } \\
\text { (HER1, HER2, HER4, EGFR) }\end{array}$ & A reversible TK
\end{tabular}

Mechanism of A highly selective blocker for the action

Clinical trial, Phase, identifier, main outcomes, author

HER2CLIMB

Phase III

NCTO2614794 15

In the tucatinib arm (tucatinib \& trastuzumab/capecitabine) versus placebo arm (trastuzumab/ capecitabine)

1-year PFS rates: 33\% versus 12\%; median PFS: 7.8 months versus 5.6 months;

2 -year OS rates: $45 \%$ versus $27 \%$; median OS: 21.9 months versus 17.4 months

Murthy et al., ${ }^{9} 2019$

A blocker of the PI3K/ AKT and MAPK signalling pathways

SUMMIT basket trial

Phase II

NCT01953926 16

Neratinib (plus fulvestrant) is clinically active in pretreated patients with HER2-mutant, HR-positive metastatic BC median PFS: 5.4 months;

ORR: 30\%;

CBR: $47 \%$

Smyth et al., ${ }^{14} 2019$ 
Table 1 continued.

\begin{tabular}{|c|c|c|c|}
\hline TKI name & Tucatinib & Neratinib & Lapatinib \\
\hline $\begin{array}{l}\text { Important clinical } \\
\text { implications }\end{array}$ & $\begin{array}{l}\text { In pretreated women with HER2- } \\
\text { positive metastatic BC (including } \\
\text { those with brain metastases), } \\
\text { adding tucatinib to a combination } \\
\text { trastuzumab/capecitabine } \\
\text { resulted in longer PFS and OS } \\
\text { than in the placebo arm; } \\
\text { compared to lapatinib and } \\
\text { neratinib, tucatinib has: } \\
\text { - A stronger activity for CNS } \\
\text { metastases } \\
\text { A better synergy with } \\
\text { trastuzumab and CHT; } \\
\text { A decreased potential for } \\
\text { EGFR-related toxicities; } \\
\text { A better tolerability that } \\
\text { increases the patient's } \\
\text { compliance; }\end{array}$ & $\begin{array}{l}\text { Synergistic effects with } \\
\text { trastuzumab in patients with } \\
\text { HER2-positive metastatic BC } \\
\text { (including those with brain } \\
\text { metastases); } \\
\text { N/C improved PFS (with a } \\
\text { trend towards improved OS) } \\
\text { versus L/C; } \\
\text { N/C contributed to a } \\
\text { delayed time to intervention } \\
\text { for symptomatic brain } \\
\text { metastases }\end{array}$ & $\begin{array}{l}\text { Penetrates into the CNS; active } \\
\text { against CNS metastases; } \\
\text { In patients with HER2-positive } \\
\text { advanced or metastatic BC } \\
\text { acts synergistically with } \\
\text { trastuzumab }\end{array}$ \\
\hline $\mathrm{AE}$ & $\begin{array}{l}\text { Diarrhoea, increased serum } \\
\text { aminotransferase levels }\end{array}$ & $\begin{array}{l}\text { Diarrhoea, neutropenia, } \\
\text { dehydration }\end{array}$ & $\begin{array}{l}\text { Diarrhoea, nausea, vomiting, } \\
\text { skin rash, fatigue }\end{array}$ \\
\hline Therapy for the AE & $\begin{array}{l}\text { Antidiarrhoeal agents } \\
\text { (loperamide, colestipol, or } \\
\text { budesonide) }\end{array}$ & $\begin{array}{l}\text { Antidiarrhoeal prophylaxis } \\
\text { with loperamide }\end{array}$ & $\begin{array}{l}\text { Antidiarrhoeal agents } \\
\text { (loperamide, colestipol, or } \\
\text { budesonide) }\end{array}$ \\
\hline
\end{tabular}

AE: adverse events; BC: breast cancer; CBR: clinical benefit rate; CHT: chemotherapy; CNS: central nervous system; HER2: human epidermal growth factor receptor-2; HR: hormone receptor; L/C: lapatinib/ capecitabine; N/C: neratinib/capecitabine; ORR: overall response rate; OS: overall survival; PFS: progression-free survival; TKI: tyrosine kinase inhibitor.

It should be emphasised that the HER2CLIMB trial was the pioneering study that involved patients with heavily pretreated HER2-positive metastatic BC (with and without CNS metastases, including those with progressive CNS metastases) (Table 1).9, ${ }^{9,5-17}$

The HER2CLIMB study has compared tucatinib plus trastuzumab/capecitabine (the tucatinib arm) to the trastuzumab/capecitabine standard therapy (the placebo arm) among patients with HER2-positive metastatic BC (Table 1). ${ }^{9}$ Notably, the HER2CLIMB trial allowed patients with CNS metastasis (approximately half of the participants had brain metastases at baseline) of any type to enrol. ${ }^{9}$ For instance, the study patients could have treated/stable or untreated brain metastases, as well as previously treated but subsequently progressing CNS metastatic lesions. ${ }^{9}$ The participants' performance status based on Eastern Cooperative Oncology Group (ECOG) was $\mathrm{O}$ or $1 .{ }^{9}$ An analysis of survival (PFS and overall survival) is presented in Table $1 .{ }^{9}$ It should be highlighted that the risk of $\mathrm{BC}$ progression or mortality in women with CNS metastases was decreased by $52 \%$ in the total HER2CLIMB trial population; 1-year PFS in the tucatinib arm was $25 \%$ versus $0 \%$ in the placebo arm, while the median PFS was 7.6 versus 5.4 months, respectively. ${ }^{9}$ The HER2CLIMB is a pioneering trial, revealing that it is possible to positively influence the survival of women with CNS metastases related to the HER2-positive BC. 
Table 2: A comparison between the monoclonal antibodies margetuximab and trastuzumab.

\begin{tabular}{|c|c|c|}
\hline Name of the antibody & Margetuximab & Trastuzumab \\
\hline Anti-HER2 antibody & \multicolumn{2}{|c|}{$\begin{array}{l}\text { A monoclonal IgG1 humanised antibody that binds the extracellular domain } \\
\text { of HER2 transmembrane receptor }\end{array}$} \\
\hline Fab & \multicolumn{2}{|c|}{ Structure, affinity and specificity for HER2 binding; } \\
\hline (similarities) & \multicolumn{2}{|c|}{ Impact on disruption of the cell signalisation (for proliferation and survival) } \\
\hline \multirow[t]{3}{*}{ Fc (differences) } & $\begin{array}{l}\text { Fc-engineered region contains five } \\
\text { different amino-acids (compared to } \\
\text { the wild-type IgG1); it expresses: }\end{array}$ & $\begin{array}{l}\text { Wild-type IgG1 immune effector } \\
\text { domain; binds and activates } \\
\text { immune cells }\end{array}$ \\
\hline & $\begin{array}{l}\text { - A higher affinity for FcyRIIIA } \\
\text { (CD16A) }\end{array}$ & \\
\hline & $\begin{array}{l}\text { - A lower affinity for FcyRIIB } \\
\text { (CD32B) }\end{array}$ & \\
\hline
\end{tabular}

Clinical trial

Phase

Identifier

Design, findings

Author, year

Unique features

$\mathrm{AE}$

Practical implications for the patients
SOPHIA

Phase III

NCTO2492711 18

Margetuximab plus CHT versus Trastuzumab plus CHT (as third-line treatment) in patients with HER2positive metastatic BC (after prior antiHER2 therapies including pertuzumab)

Rugo et al., ${ }^{10} 2019$

Fc-engineered region activates immune response; Fc portion tightly binds to the Fc receptors and stimulates strong ADCC (e.g., in patients with low-affinity Fc receptors); a possibility of combination with immunotherapy or CHT; a potential treatment role in earlier stages of BC

Infusion reactions

Margetuximab plus CHT improves PFS (in third-line treatment) compared to Trastuzumab plus CHT; PFS benefits are more expressed in low-affinity CD16A-158F allele carriers (FF or $\mathrm{FV}$ ) than in high-affinity ones (VV); In contrast, the $\mathrm{VV}$ carriers respond better to trastuzumab
The Retreatment after HErceptin

Adjuvant trial

NCTO0475670 19

Trastuzumab plus a taxane as first-line treatment in patients with metastatic BC (who had relapsed after adjuvant trastuzumab for HER2-positive early BC) (median PFS: 8 months; median OS: 25 months)

Láng et al.,20 2014

A monoclonal antibody that binds to the HER2 and inhibits the proliferation of cells overexpressing HER2 protein

Alopecia, diarrhoea, risk of cardiac toxicity (e.g., LVEF decline, CHF), haematologic deficiencies

Trastuzumab, in combination with $\mathrm{CHT}$ (a taxane), is an effective and well-tolerated first-line treatment for HER2-positive metastatic $B C$, in patients who relapsed after trastuzumabbased adjuvant therapy

AE: adverse events; ADCC: antibody-dependent cell-mediated cytotoxicity; BC: breast cancer; CHF: congestive heart failure; HER2: human epidermal growth factor receptor-2; CHT: chemotherapy; LVEF: left ventricular ejection fraction; OS: overall survival; PFS: progression-free survival. 
With regard to the safety concerns, diarrhoea, increased aminotransferase levels, palmar-plantar erythrodysesthesia syndrome, nausea, vomiting, and fatigue were common adverse effects in the tucatinib-combination (trastuzumab/ capecitabine) group. ${ }^{9}$ A comparison of tucatinib with the other TKI (e.g., lapatinib and neratinib) is presented in Table 1.,9,13

\section{MARGETUXIMAB: A UNIQUE OPPORTUNITY FOR PATIENTS WITH BREAST CANCER HARBOURING GENETIC ALTERATIONS IN THE ANTIBODY FRAGMENT CRYSTALLISABLE REGION}

Margetuximab is a unique anti-HER2 monoclonal antibody, which gives new hope that immunotherapy can bring some advantages to patients with HER2-positive BC. ${ }^{10}$ Moreover, it may initiate a certain way of selecting patients, according to their type of fragment crystallisable (Fc) immune receptors (low versus high-affinity), possibly via introducing a genetic test which can facilitate a choice of therapeutic agent (e.g., margetuximab versus trastuzumab). ${ }^{10}$ Margetuximab is considered a modified version of trastuzumab which, in addition to inhibiting HER2 signalling, stimulates the immune system to attack the HER2-positive BC cells. ${ }^{10}$ This is mediated via antibody-dependent cellular cytotoxicity (ADCC), and occurs when the FC region of the trastuzumab antibody binds to the Fc receptors on natural killer cells and other immune effectors. ${ }^{10}$ Margetuximab has been engineered with the Fc portion of the antibody that allows it to bind more tightly to the Fc receptors. This ability is critically important, because many patients display a polymorphism in their Fc receptors, resulting in poor binding to the antibodies, which in turn can impair therapeutic effects (Table 2).10,18-20 It should be emphasised that margetuximab represents an Fc-optimised anti-HER2 antibody, which has an augmented affinity for CD16a and a decreased affinity for CD32B. ${ }^{10}$ The purpose of this affinity modification is to augment host immunity (innate and adaptive). ${ }^{10}$ The SOPHIA trial has compared the therapeutic effects between margetuximab (in combination with $\mathrm{CHT}$ ) and trastuzumab (in combination with $\mathrm{CHT}$ ), and enrolled patients with HER2-positive advanced or metastatic
BC who had received prior treatment with trastuzumab, pertuzumab, and T-DM1 (in the third-line setting) (Table 2)..$^{10}$ The SOPHIA study population resembled that of the HER2CLIMB trial, apart from the patients with progressive CNS metastases, and including patients with stable CNS metastases. ${ }^{9,10}$

According to the results of the SOPHIA trial, in the group with the CD16a $F$ allele (low-affinity), approximately a 4-month difference in the median OS has been reported..$^{10}$ In contrast, when evaluating the group of patients with the CD16a $V$ allele (high-affinity), this trend was reversed. ${ }^{10}$ However, it should also be noted that in the SOPHIA study, the subpopulation with highaffinity Fc receptors was very limited, and such patients had different characteristics compared to the majority of the trial population. ${ }^{10}$ Furthermore, the patients in the margetuximab arm were usually more heavily pretreated and had more metastatic lesions (e.g., hepatic and CNS). ${ }^{10}$ At this point in time, it appears that the women with the CD16a $F$ allele have achieved greater benefits from the application of margetuximab. ${ }^{10}$

The main unanswered question is whether or not the use of margetuximab should be linked to the patient's genotype (e.g., should a genetic test be used to verify the presence of the CD16a $F$ allele). In addition, the findings from the SOPHIA trial have provided an important clue that immunotherapy offers valuable clinical potential in the HER2-positive BC setting. ${ }^{10}$ Moreover, since the SOPHIA trial has included a group of heavily pretreated patients, it is conceivable that if the benefits in such patients have been driven by immune mechanisms, potentially even larger benefits in previously untreated patients with HER2-positive BC may be expected. However, this would require validation in future trials. ${ }^{10}$ Additionally, margetuximab can be used in combination with other anticancer agents. In particular, because of its ability to stimulate intense ADCC, margetuximab may work in concert with immunotherapy (e.g., immune checkpoint inhibitors) or $\mathrm{CHT}$ (e.g., anthracyclines).$^{10}$ Margetuximab is well-tolerated, with a toxicity profile similar to trastuzumab, with one exception relevant to more frequent infusion-related reactions (Table 2). ${ }^{10}$ 
Table 3: A comparison between the antibody drug-conjugates trastuzumab deruxtecan (T-DXd) and trastuzumab emtansine (T-DM1).

\begin{tabular}{lll}
\hline $\begin{array}{l}\text { Similarities and differences of } \\
\text { the ADC }\end{array}$ & $\begin{array}{ll}\text { Trastuzumab deruxtecan } \\
\text { (T-DXd; DS-8201) }\end{array}$ & $\begin{array}{l}\text { Trastuzumab emtansine } \\
\text { (T-DM1) }\end{array}$
\end{tabular}

Antibody class

Trastuzumab: an anti-HER2 IgG1 humanised monoclonal antibody (an identical part)

Payload

Linker

Drug: Antibody ratio

Mechanism of action

Clinical trial

Phase

Identifier

Main outcomes

Author, year

Important clinical benefits

AE

Therapy for the AE
Topoisomerase I inhibitor

(exatecan derivative) high potency, membrane-permeable, short systemic half-life

Tumour-selective, cleavable, stable bond linker-payload

7:8 (high)

Inhibition of topoisomerase 1; bystander killing effect in the $\mathrm{BC}$ tumour tissue

DESTINY-BreastO1

Phase II

NCTO3248492 22

In the T-DXd arm:

ORR: $60.3 \%$;

Median PFS: 16.4 months

Modi et al.," 2019

Durable antitumour activity in pretreated (with T-DM1) patients with HER2-positive metastatic BC; a broader anti-tumour activity than T-DM1

Potentially serious ILD or pneumonitis; nausea, vomiting, fatigue, hair loss, and myelosuppression (anaemia, neutropenia)

Corticosteroids for ILD (required close monitoring for pulmonary symptoms)
Anti-tubulin (DM1)

(maytansine derivative) a

microtubule inhibitor

Covalently linked to trastuzumab

\section{3:5 (low)}

DM1 (a potent cytotoxic agent that inhibits microtubules) is selectively delivered to the HER2-positive $\mathrm{BC}$ cells

EMILIA

Phase III

\section{NCT00829166 23}

Median PFS: 9.6 months with T-DM1 versus 6.4 months with lapatinib/capecitabine;

Median OS: 29.9 months with T-DM1 versus 25.9 months with lapatinib/capecitabine

Diéras et al., ${ }^{24} 2017$

T-DM1 significantly prolonged PFS and OS (with less toxicity than lapatinib/capecitabine) in patients with HER2-positive advanced BC previously treated with trastuzumab and a taxane

Thrombocytopenia, increased serum aminotransferase levels, diarrhoea, nausea, vomiting, and palmarplantar erythrodysesthesia

As required, depending on symptoms

ADC: antibody drug-conjugate; AE: adverse events; BC: breast cancer; HER2: human epidermal growth factor receptor 2; CHT: chemotherapy; ILD: interstitial lung disease; OS: overall survival; PFS: progression-free survival; ORR: overall response rate; T-DM1: trastuzumab emtansine; T-DXd: trastuzumab deruxtecan. 


\section{TRASTUZUMAB DERUXTECAN: A NEW GENERATION ANTIBODY-DRUG CONJUGATE AGAINST HER2-POSITIVE BREAST CANCER}

T-DXd exemplifies a powerful ADC against HER2 that is composed of an anti-HER2 antibody, a cleavable tetrapeptide-based linker, and a payload in a form of the cytotoxic topoisomerase I inhibitor. ${ }^{21}$

The main similarities and differences between T-DXd and T-DM1 are summarised in Table 3. 11,22-24 It is important to keep in mind that the payload of T-DXd (a topoisomerase I inhibitor) represents a different type of $\mathrm{CHT}$ compared to the one typically applied in patients with HER2-positive BC. This design can contribute to less resistance to such CHT, especially because there are more payload particles per antibody. Moreover, the payload is able to diffuse from the targeted HER2-positive cells and damage the neighbouring tumour cells, causing a desirable 'bystander killing effect.'11,15,25 The DESTINYBreast01 (Phase II) trial has investigated T-DXd involved patients with HER2-positive metastatic $\mathrm{BC}$, who had received on average six prior treatments (including trastuzumab, pertuzumab, and T-DM1). ${ }^{11}$ Notably, the objective response rate was approximately $61 \%$, the duration of response was almost 15.0 months, and the median PFS was 16.4 months. ${ }^{11}$ Moreover, approximately 13\% of participants in this trial had previously treated CNS metastases. It should be underscored that PFS was 18 months." This finding suggests that the prior treatment for CNS metastases had not reduced the favourable effects of subsequent T-DXd use.

Adverse effects of T-DXd (e.g., nausea, vomiting, fatigue, hair loss, and neutropenia, which was usually afebrile) were mild. ${ }^{11}$ Also, it is encouraging that no clinically significant cardiotoxic effects were reported. However, the most severe treatment-emergent adverse events were interstitial lung disease (ILD) and pneumonitis that developed in $13.6 \%$ of the participants (e.g., usually low grade, but in $2.2 \%$ it was fatal). ${ }^{1}$ Because of potential lung toxicity, patients treated with T-DXd have to be monitored very closely for ILD." In order to mitigate severe respiratory complications, lung scans have been introduced for early detection of any suspicious pulmonary abnormalities. This would make it possible to adjust the dose or terminate the T-DXd therapy and, simultaneously, implement the therapy with corticosteroids." In addition, other questions to be answered by future studies are relevant to the potential use of T-DXd earlier in the BC course (e.g., in the first-line therapy or earlystage BC) or its possible synergistic actions with some other anticancer therapies in the adjuvant and metastatic setting (e.g., agents that spare the lymphocytes or target DNA repair).11,25 Also, future randomised trials are needed to address the magnitude of benefit of the T-DXd therapy compared to the current standard of care.

\section{CONCLUSIONS}

In patients who had progressed even after multiple lines of previous therapy, HER2 as a therapeutic target is still a valid choice. Moreover, recent clinical studies on new therapeutics (e.g., tucatinib, margetuximab, and T-DXd) for patients with HER2-positive metastatic BC have brought some good news for this patient population and their treatment teams. Tucatinib (a selective HER2 TKI) added to trastuzumab/ capecitabine combination has improved PFS and OS (compared to placebo and the above combination) in the population of previously treated women with HER2-positive metastatic $\mathrm{BC}$, including those with brain metastases. Similarly, it is expected that margetuximab (Fc domain-engineered anti-HER2 antibody) may represent an innovative therapeutic strategy for patients with HER2-positive metastatic BC, who are low-affinity CD16a-158F allele carriers (FF or $F V$ ). In addition, margetuximab may play a role in the earlier stages of BC therapy. However, this possibility needs to be examined in detail in future trials.

Likewise, it should be noted that T-DXd, representing a new generation ADC against HER2, has revealed remarkable anti-tumour activity in patients with HER2-positive metastatic BC, who were heavily pretreated. However, because of some safety concerns such as ILD or pneumonitis, which pose serious risks, vigilant monitoring for signs and symptoms of ILD and immediate therapeutic intervention (e.g., glucocorticoids) are mandatory for the management of such patients. Nevertheless, it is expected that T-DXd may become a 'new standard of care' for patients with advanced or metastatic HER2-positive BC. 
Some urgent questions for further research on TKI and ADC in this area are mostly related to the level of clinical benefits of these agents in specific clinical contexts, and the possibilities of their combined applications with other medications in patients with HER2-positive metastatic BC, especially those with progressive brain metastases. Furthermore, studies aimed at detection of predictive and prognostic biomarkers, to guide the individualised diagnostic work-up and targeted treatment of women with HER2-positive BC, represent research priorities. Simultaneously, there is a need for further studies to investigate and validate innovative therapeutics in the most challenging HER2positive $\mathrm{BC}$ management setting.

\section{References}

1. Arteaga C et al. Treatment of HER2positive breast cancer: current status and future perspectives. Nat Rev Clin Oncol. 2012;9:16-32

2. Beser AR et al. HER-2, TOP2A and chromosome 17 alterations in breast cancer. Pathol Oncol Res. 2007:13:180-5.

3. Maennling AE et al. Molecular targeting therapy against EGFR family in breast cancer: progress and future potentials. Cancers (Basel). 2019;11(12): pii: E1826.

4. Nielsen DL et al. Efficacy of HER2targeted therapy in metastatic breast cancer. Monoclonal antibodies and tyrosine kinase inhibitors. Breast. 2013;22(1):1-12.

5. Krop IE et al. Trastuzumab emtansine versus treatment of physician's choice in patients with previously treated HER2-positive metastatic breast cancer (TH3RESA): final overall survival results from a randomised open-label Phase 3 trial. Lancet Oncol. 2017;18(6):743-54.

6. Wang J, Xu B. Targeted therapeutic options and future perspectives for HER2-positive breast cancer. Sig Transduct Target Ther. 2019;4(34): doi:10.1038/s41392-019-0069-2.

7. National Comprehensive Cancer Network (NCCN) Guidelines ${ }^{\oplus}$. Breast Cancer (Version 3.2019). 2019. Available at: https://www.nccn.org/ professionals/physician gls/pdf/ breast.pdf. Last accessed: December 10, 2019.

8. Yuan P, Gao SL. Management of breast cancer brain metastases: focus on human epidermal growth factor receptor 2-positive breast cancer. Chronic Dis Transl Med. 2017;3(1):2132: doi: 10.1016/j.cdtm.2017.01.004.

9. Murthy RK et al. Tucatinib, trastuzumab, and capecitabine for HER2-positive metastatic breast cancer. N Engl J Med. 2019; doi: 10.1056/NEJMoa1914609.

10. Rugo HS et al. SOPHIA primary analysis: a Phase 3 (P3) study of margetuximab $(M)+$ chemotherapy
(C) versus trastuzumab ( $T$ ) $+C$ in patients (pts) with HER2+ metastatic (met) breast cancer (MBC) after prior anti-HER2 therapies (Tx). J Clin Oncol. 2019;37(Suppl 15): 1000.

11. Modi S et al. DESTINY-BreastO1 Investigators. trastuzumab deruxtecan in previously treated HER2-positive breast cancer. N Engl J Med. 2019; doi: 10.1056/ NEJMoa1914510.

12. Verma $\mathrm{S}$ et al. Trastuzumab emtansine for HER2-positive advanced breast cancer. $N$ Engl J Med. 2012;367:1783-91.

13. Saura $\mathrm{C}$ et al. Neratinib + capecitabine versus lapatinib + capecitabine in patients with HER2+ metastatic breast cancer previously treated with $\geq 2$ HER2 directed regimens: findings from the multinational, randomized, Phase III NALA trial. J Clin Oncol. 2019;37(Suppl 15):1002

14. Smyth LM et al. Update on the phase II SUMMIT trial: neratinib + fulvestrant for HER2-mutant, HR-positive, metastatic breast cancer. Ann Oncol. 2019;30(Suppl 3):iii1-26.

15. Seattle Genetics, Inc. A study of tucatinib vs. placebo in combination with capecitabine \& trastuzumab in patients with advanced HER2+ breast cancer (HER2CLIMB). NCTO2614794. https://clinicaltrials.gov/ct2/show/ NCTO2614794.

16. Puma Biotechnology, Inc. Neratinib HER Mutation Basket Study (SUMMIT) (SUMMIT). NCT01953926. https://clinicaltrials.gov/ct2/show/ NCT01953926.

17. Puma Biotechnology, Inc. A study of neratinib plus capecitabine versus lapatinib plus capecitabine in patients with HER2+ metastatic breast cancer who have received two or more prior HER2 directed regimens in the metastatic setting (NALA). NCT01808573. https://clinicaltrials. gov/ct2/show/NCT01808573.

18. MacroGenics. Margetuximab plus chemotherapy vs trastuzumab plus chemotherapy in the treatment of
HER2+ metastatic breast cancer (SOPHIA). NCTO2492711. https:// clinicaltrials.gov/ct2/show/ NCTO2492711.

19. Hoffmann-La Roche. A Study of herceptin (trastuzumab) in women with metastatic breast cancer. NCTO0475670. https://clinicaltrials. gov/ct2/show/NCTO0475670.

20. Láng | et al. Trastuzumab retreatment after relapse on adjuvant trastuzumab therapy for human epidermal growth factor receptor 2-positive breast cancer: final results of the retreatment after HErceptin adjuvant trial. Clin Oncol (R Coll Radiol). 2014;26(2):81-9.

21. Tamura $\mathrm{K}$ et al. Trastuzumab deruxtecan (DS-8201a) in patients with advanced HER2-positive breast cancer previously treated with trastuzumab emtansine: a doseexpansion, Phase 1 study. Lancet Oncol. 2019;20(6):816-26.

22. Daiichi Sankyo, Inc. A study of DS 8201a in metastatic breast cancer previously treated with trastuzumab emtansine (T-DM1). NCT03248492. https://clinicaltrials.gov/ct2/show/ NCTO3248492.

23. Hoffmann-La Roche. A study of trastuzumab emtansine versus capecitabine + lapatinib in participants with HER2-positive locally advanced or metastatic breast cancer (EMILIA). NCT00829166. https://www.clinicaltrials.gov/ct2/ show/NCTO0829166.

24. Diéras $\vee$ et al. Trastuzumab emtansine versus capecitabine plus lapatinib in patients with previously treated HER2-positive advanced breast cancer (EMILIA): a descriptive analysis of final overall survival results from a randomised, open-label, Phase 3 trial. Lancet Oncol. 2017;18(6):732-42.

25. Trail PA et al. Antibody drug conjugates for treatment of breast cancer: novel targets and diverse approaches in ADC design. Pharmacol Ther. 2018;181:126-42. 